\title{
Tratamiento con fármacos antiangiogénicos de la degeneración macular asociada a la edad exudativa: análisis de la función visual a largo plazo en la práctica clínica habitual
}

\section{Treatment of exudative age-related macular degeneration with anti-angiogenic drugs: analysis of long-term visual function in routine clinical practice}

\author{
Ana Palazón-Cabanes ${ }^{1 *}$, Begoña Palazón-Cabanes² e Inmaculada Sellés-Navarro ${ }^{1}$ \\ ${ }^{1}$ Servicio de Oftalmología, Hospital General Universitario Reina Sofía; ${ }^{2}$ Servicio de Neurología, Hospital Universitario Virgen de la Arrixaca. Murcia, \\ España
}

\section{Resumen}

Objetivo: Analizar los resultados visuales a largo plazo de ojos con degeneración macular asociada a la edad exudativa (DMAE) tratados con inhibidores del factor de crecimiento endotelial en la práctica clínica real. Método: Estudio retrospectivo que incluye 48 ojos de 44 pacientes con DMAE húmeda, únicamente tratados con fármacos antiangiogénicos. Se analizaron los valores de agudeza visual (AV) tras 6 meses, y tras 1, 3 y 5 años de seguimiento. Resultados: La AV mejoró de 61.04 a 65.83 letras a los 6 meses, con una pérdida de -1.14, -16.88 y -27.50 letras respecto a la AV inicial a los 1, 3 y 5 años, respectivamente. El porcentaje de ojos con una pérdida $<15$ letras fue del 50 y del 33.3\% a los 3 y 5 años, respectivamente. Un 14.6\% mejoró $\geq$ 15 letras a los 3 años y un $12.5 \%$ a los 5 años. Los ojos con $A V \geq 70$ letras previo al tratamiento fueron el $41.7 \%$, descendiendo al $22.92 \%$ a los 3 años y a $10.42 \%$ a los 5 años. El número medio de inyecciones fue $4.81,2.21,1.73,1.17$ y 0.9 en el primer, segundo, tercer, cuarto y quinto años de seguimiento, respectivamente, con un número medio de visitas de 7.19, 4.88, 4.02, 3.40 y 2.85, respectivamente. La edad avanzada, la menor AV basal y la pseudofaquia presentaron resultados visuales menos satisfactorios a largo plazo. Conclusiones: Los resultados visuales de este estudio son inferiores a estudios previos. Sin embargo, el número de inyecciones recibidas fue menor y todos los ojos completaron un seguimiento de 5 años.

Palabras clave: Degeneración macular asociada a la edad. Inhibidores del factor de crecimiento endotelial. Agudeza visual. Función visual. Resultados a largo plazo.

\section{Abstract}

Purpose: To analyze the long-term visual outcomes of vascular endothelial growth factor inhibitors in treatment-naïve eyes with neovascular age-related macular degeneration (AMD). Methods: Retrospective study with 48 eyes of 44 treatment-naïve patients with neovascular AMD treated only with anti-angiogenic. Visual acuity (VA) results were analyzed after 6 months and 1,3, and 5 years of follow-up. Results: Mean VA improved from 61.04 to 65.83 letters after 6 months, with a loss of -1.14, -16.88 and -27.50 letters compared to baseline after 1, 3 and 5 years. The proportion of eyes that lost $<15$ letters at 3 and 5 years was $50 \%$ and $33.3 \%$, respectively. The proportion of eyes with a gain of $>15$ letters was $14.6 \%$ and $12.5 \%$ at 3 and

Fecha de recepción: 16-06-2019 Fecha de aceptación: 09-06-2020 Email: a.palazoncabanes@gmail.com DOI: 10.24875/RMO.M20000124
Disponible en internet: 01-09-2020 Rev Mex Oftalmol. 2020;94(5):213-220

www.rmo.com.mx (http://creativecommons.org/licenses/by-nc-nd/4.0/) 
5 years. The proportion of eyes with a VA $>70$ letters was $41.7 \%$ at baseline and $22.92 \%$ and $10.42 \%$ after 3 and 5 years. The mean number of injections received was 4.81, 2.21, 1.73, 1.17 and 0.9 at years 1, 2, 3, 4, and 5, respectively, and the mean number of visits was 7.19, 4.88, 4.02, 3.40, and 2.85. Older age, lower VA at baseline and pseudophakic eyes showed worse long-term visual outcomes. Conclusions: The visual outcomes of this study are inferior compared to previous studies. Nevertheless, the number of injections received was lower, and all eyes completed a 5-year follow-up.

Key words: Age-related macular degeneration. Endothelial growth factor inhibitors. Visual acuity. Visual function. Long-term outcomes.

\section{Introducción}

La degeneración macular asociada a la edad (DMAE) es la principal causa de pérdida de visión central irreversible y de ceguera legal entre los individuos mayores de 55 años en países desarrollados, y se estima que afectará a más de 3 millones de personas en los EE.UU. en 2020. La aparición de los fármacos antiangiogénicos, dirigidos contra el factor de crecimiento vascular endotelial (VEGF) ha supuesto una revolución en el tratamiento de la DMAE exudativa'.

El bevacizumab (Avastin ${ }^{\circledR}$, Genentech) es una molécula de anticuerpo completa que reconoce e inhibe todas las isoformas del VEGF-A humano. Se pensó que su alto peso molecular era una limitación para penetrar en la retina ${ }^{2}$, y esto promovió el desarrollo de un fragmento Fab humanizado del mismo anticuerpo: el ranibizumab (Lucentis ${ }^{\circledR}$, Genentech), que fue aprobado por la FDA (Food and Drug Administration, por sus siglas en inglés) en el año 2006 a raíz de los estudios pivotales MARINA ${ }^{3}$ y ANCHOR ${ }^{4}$. Posteriormente, el estudio CATT $^{5}$ demostró que la eficacia del ranibizumab y el bevacizumab era equivalente en términos de AV.

Tras estos estudios, surgió la importante cuestión de cómo conseguir los mayores beneficios en términos de ganancia visual y su mantenimiento con el menor número de inyecciones. Con este objetivo, se diseñaron estudios con regímenes de tratamiento a intervalos fijos $^{6,7}$, pero mostraron resultados globales menos favorables que los regímenes mensuales. Más tarde, otras investigaciones estudiaron los posibles beneficios de un régimen a demanda, denominado "Pro Re Nata» (PRN) ${ }^{8-11}$, el cual consistía en una dosis inicial de tres inyecciones mensuales consecutivas de ranibizumab, seguidas de visitas mensuales y retratamientos en función de los criterios funcionales y/o estructurales establecidos en cada uno de los estudios. Este régimen demostró mantener la eficacia del tratamiento. Posteriormente, se propuso un nuevo sistema denominado «Treat and Extend» (T\&E) ${ }^{12}$, en el cual, los intervalos de retratamiento se van incrementando secuencialmente en 2 semanas hasta un máximo de 12 semanas y, en caso de recurrencia de los signos de exudación, se comenzaría con el seguimiento a intervalos más estrechos para ir nuevamente espaciándolos de manera progresiva. Con este régimen se consigue una mejora significativa de la $\mathrm{AV}$ con un número menor de visitas e inyecciones en comparación con los protocolos mensuales.

El último antiangiogénico introducido para el tratamiento de la DMAE neovascular es el aflibercept (Eylea $^{\circledR}$ Regeneron Pharmaceuticals Inc), con una capacidad de unión al VEGF casi 100 veces superior que el ranibizumab y el bevacizumab, y con una eficacia similar al ranibizumab, documentada en dos importantes ensayos clínicos (VIEW 1 y VIEW 2) ${ }^{13,14}$, y requiere un menor número de inyecciones.

\section{Material y métodos}

Estudio descriptivo y retrospectivo en el que se han recogido datos de un total de 48 ojos de 44 pacientes, incluidos entre los años 2008 y 2011, con diagnóstico de DMAE exudativa sin tratamiento previo ocular y que iniciaron terapia antiangiogénica intravítrea tras el diagnóstico de su enfermedad macular. Todos los pacientes completaron un periodo de seguimiento de al menos 5 años. Se han recogido y estudiado los valores de AV (escala ETDRS) a 6 meses, 1, 3 y 5 años de seguimiento. Además, se ha analizado la evolución de la función visual según las distintas variables clínicas: AV basal previa al tratamiento antiangiogénico, características demográficas (edad y sexo), si se trataba del primer o segundo ojo afectado o bien si el ojo estudiado era fáquico o pseudofáquico. Por último, se sometió a contraste las AV documentadas en los diferentes tiempos respecto a cada una de estas variables clínicas.

\section{Resultados}

\section{Agudeza visual}

La media de la AV basal fue de $61.04 \pm 2.26$ letras (IC 95\%: 56.49-65.59). A los 3 meses de seguimiento 
se observa una mejoría de la AV a 66.15 letras (SD, 1.79 letras), que se mantuvo sin cambios a los 6 meses de seguimiento con una media de 65.83 letras (SD, 1.73 letras). Sin embargo, la media de AV sufrió un descenso progresivo en los siguientes tiempos valorados, siendo de 59.90 letras (SD, 2.99 letras), 44.17 letras (SD, 3.88 letras) y 33.54 letras (SD, 3.89 letras) a los 1,3 y 5 años de seguimiento, respectivamente. Estos datos revelan una pérdida de visión respecto al momento del diagnóstico de 16.88 letras (SD, 4.09 letras) y de 27.50 letras (SD, 4.36 letras), tras un periodo de 3 y 5 años de terapia antiangiogénica, respectivamente (Fig. 1).

Los ojos que sufrieron una pérdida de $\mathrm{AV}>15$ letras, respecto a la $A V$ inicial, fue el $50 \%$ a los 3 años y el $66.7 \%$ a los 5 años de seguimiento, respectivamente. Por otro lado, se objetivó una mejoría de la AV > 15 letras en un 14.6 y un $12.5 \%$ de los casos a los 3 y 5 años, respectivamente.

\section{Terapia antiangiogénica}

El $45.8 \%$ de los casos fue tratado con un único tipo de fármaco: el $13.6 \%$ solo con bevacizumab y el $86.4 \%$ solo con ranibizumab. Un $54.2 \%$ de los casos recibió más de un tipo de fármaco. Entre estos últimos, se empleó bevacizumab en un $48.37 \%$ de las inyecciones, ranibizumab en un $44.48 \%$ de las inyecciones y aflibercept en un $7.14 \%$ de las inyecciones.

En la tabla 1 se recogen el número medio de inyecciones intravítreas de fármacos anti-VEGF, así como el número medio de visitas al año en cada uno de los años de seguimiento.

\section{Evolución de la agudeza visual en función de otras variables clínicas}

\section{Agudeza visual basal}

Los pacientes con una $\mathrm{AV}$ en el momento del diagnóstico $\geq 70$ letras $(20 / 40)(n=20)$ experimentaron una pérdida de 24.75 letras (SD, 6.37) y de 39.25 letras (SD, 6.90) a los 3 y 5 años de seguimiento, respectivamente. Los casos que inicialmente tenían una visión $\leq 35$ letras $(20 / 200)(n=5)$ experimentaron una mejoría de la AV en 8 letras (SD, 7.00) a los 3 años de seguimiento. A los 5 años, el balance fue de una pérdida de 4 letras respecto a la AV basal en estos ojos (Fig. 1).

La proporción de ojos que experimentó una pérdida de $A V>15$ letras a los 3 años de seguimiento fue del $55 \%$ para AV basales $\geq 70$ letras, del $52.2 \%$ para los
Tabla 1. Número medio y desviación estándar de inyecciones intravítreas de fármaco antiangiogénico y de visitas médicas al año durante un periodo de seguimiento de 5 años

\begin{tabular}{|c|c|c|c|c|c|}
\hline & 1. er año & $2^{\circ}$ ลก๊̃o & $3^{\text {er }}$ aก̃o & 4. ${ }^{\circ}$ aก̃o & $5^{\circ}$ aก̃o \\
\hline $\begin{array}{l}\text { N. }{ }^{\circ} \text { inyecciones/ } \\
\text { año }\end{array}$ & $\begin{array}{c}4.81 \pm \\
0.16\end{array}$ & $\begin{array}{c}2.21 \pm \\
0.23\end{array}$ & $\begin{array}{l}1.73 \pm \\
0.25\end{array}$ & $\begin{array}{c}1.17 \pm \\
0.23\end{array}$ & $\begin{array}{l}0.9 \pm \\
0.21\end{array}$ \\
\hline N. ${ }^{0}$ visitas/año & $\begin{array}{c}7.19 \pm \\
0.23\end{array}$ & $\begin{array}{c}4.88 \\
\pm 0.26\end{array}$ & $\begin{array}{c}4.02 \pm \\
0.22\end{array}$ & $\begin{array}{c}3.40 \pm \\
0.25\end{array}$ & $\begin{array}{c}2.85 \pm \\
0.25\end{array}$ \\
\hline
\end{tabular}

que veían entre 36-69 letras y del 20\% para los que la AV era $\leq 35$ letras. A los 5 años de seguimiento, esta proporción fue del 80, 60.9 y $40 \%$ para cada uno de los grupos, respectivamente. Los ojos con buena función visual al diagnóstico ( $\geq 70$ letras) no alcanzaron una ganancia visual significativa a largo plazo (ningún caso muestra una mejoría > 15 letras). En contraste, los ojos con una pobre visión inicial ( $\leq 35$ letras) ganaron $>15$ letras en un 60 y un $20 \%$ a los 3 y 5 años, respectivamente.

Los ojos que leían un mayor número de letras en el momento del debut de la neovascularización coroidea mantenían una AV superior al resto de casos a lo largo de tiempo, siendo esta diferencia significativa al año de seguimiento $(p=0.018)$. Los ojos con peores AV iniciales tienden a mantener AV más bajas que el resto, aunque estos resultados no fueron estadísticamente significativos (Tabla 2).

\section{EDAD}

La edad media de los pacientes fue de $76.75 \pm 0.73$ años (IC 95\%: 75.28-78.22). Los datos muestran una pérdida de 22.33 letras (SD, 5.52) y 31.83 letras (SD, 5.80) en los casos con una edad $\geq 75$ años $(n=30)$ a los 3 y 5 años de seguimiento, respectivamente. Para pacientes $<75$ años $(n=18)$, la pérdida de AV fue de 7.78 letras (SD, 5.40) y de 20.28 letras (SD, 6.33) a los 3 y 5 años de seguimiento, respectivamente (Fig. 1). La progresión del proceso neovascular resultó ser más tórpida en los ojos de los pacientes de mayor edad. Sin embargo, estas diferencias solo fueron estadísticamente significativas a los 3 años de seguimiento $(p=0.020)$ (Tabla 2).

\section{SEXo}

El $39.6 \%$ eran varones y el $60.4 \%$ mujeres. En pacientes varones, se obtuvo una pérdida de 24.47 letras (SD, 7.14) a los 3 años y de 32.37 letras (SD, 


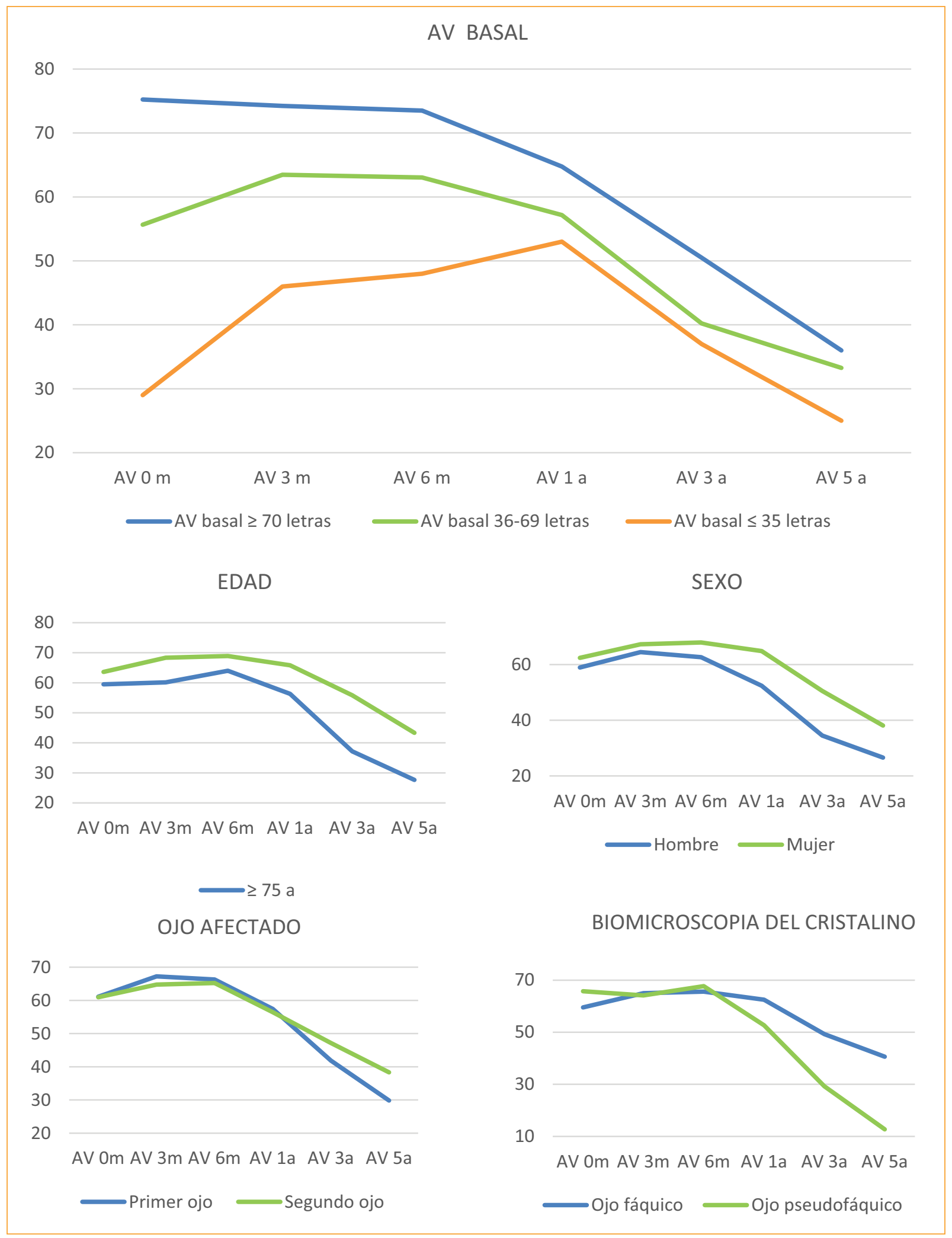

Figura 1. Representación gráfica de la evolución de la agudeza visual (AV) en el tiempo de ojos con degeneración macular asociada a la edad exudativa tratados con inyecciones intravítreas de fármacos antiangiogénicos, según las distintas variables clínicas. 
Tabla 2. Análisis bivariado (test no paramétrico U de Mann-Whithney) de los resultados visuales según las distintas variables clínicas

\begin{tabular}{|c|c|c|c|c|c|c|c|}
\hline \multicolumn{3}{|c|}{ Agudeza visual basal } & \multirow[t]{2}{*}{ Valor p } & \multicolumn{3}{|c|}{ Edad } & \multirow[t]{2}{*}{ Valor $\mathbf{p}$} \\
\hline & $<70$ letras & $\geq 70$ letras & & & $<75$ años & $\geq 75$ años & \\
\hline 1 año & $56.43 \pm 19.57$ & $64.75 \pm 21.85$ & $0.018^{* *}$ & 1 año & $65.83 \pm 19.65$ & $56.33 \pm 20.88$ & $0.058^{*}$ \\
\hline 3 años & $39.64 \pm 25.85$ & $50.50 \pm 27.81$ & 0.118 & 3 años & $55.83 \pm 22.44$ & $37.17 \pm 27.31$ & $0.020^{* *}$ \\
\hline \multirow[t]{3}{*}{5 años } & $31.79 \pm 24.54$ & $36.00 \pm 30.59$ & 0.624 & 5 años & $43.33 \pm 23.94$ & $27.67 \pm 27.54$ & $0.052^{*}$ \\
\hline & \multirow{2}{*}{$\leq 35$ letras } & \multirow{2}{*}{$>35$ letras } & & \multicolumn{3}{|c|}{ Sexo } & \multirow[t]{2}{*}{ Valor $\mathrm{p}$} \\
\hline & & & & & Hombre & Mujer & \\
\hline 1 año & $53.00 \pm 10.95$ & $60.70 \pm 21.54$ & $0.062^{*}$ & 1 año & $52.37 \pm 27.05$ & $64.83 \pm 13.72$ & 0.211 \\
\hline 3 años & $37.00 \pm 14.41$ & $45.00 \pm 28.03$ & 0.275 & 3 años & $34.47 \pm 27.93$ & $50.53 \pm 24.73$ & $0.055^{*}$ \\
\hline 5 años & $25.00 \pm 15.41$ & $34.53 \pm 27.98$ & 0.449 & 5 años & $26.58 \pm 22.80$ & $38.10 \pm 28.89$ & 0.179 \\
\hline \multicolumn{3}{|c|}{ Ojo afectado } & \multirow[t]{2}{*}{ Valor $\mathbf{p}$} & & \multicolumn{2}{|c|}{ Biomicroscopia del cristalino } & \multirow[t]{2}{*}{ Valor $p$} \\
\hline & Primero & Segundo & & & Fáquico & Pseudofáquico & \\
\hline 1 año & $57.41 \pm 24.47$ & $63.10 \pm 14.62$ & 0.842 & 1 año & $62.50 \pm 18.76$ & $52.69 \pm 25.30$ & 0.244 \\
\hline 3 años & $41.85 \pm 29.91$ & $47.14 \pm 22.94$ & 0.706 & 3 años & $49.26 \pm 23.90$ & $29.23 \pm 30.34$ & $0.048^{* *}$ \\
\hline 5 años & $29.81 \pm 28.54$ & $38.33 \pm 24.72$ & 0.266 & 5 años & $40.59 \pm 24.33$ & $12.69 \pm 23.06$ & $0.001^{* *}$ \\
\hline
\end{tabular}

*Valores muy cercanos a $\mathrm{p}<0.05$

**Significación estadística.

6.95) a los 5 años, mientras que, en mujeres, la pérdida fue de 11.90 letras (SD, 4.78) y 24.31 letras (SD, 5.63) a los 3 y 5 años de seguimiento, respectivamente (Fig. 1). El sexo femenino presenta una fuerte tendencia a mantener una mayor AV a lo largo del tiempo, sin ser estas diferencias estadísticamente significativas (Tabla 2).

\section{Ojo AFECTAdo}

Un $56.3 \%$ de los casos correspondían al primer ojo con desarrollo de neovascularización coroidea y un $43.7 \%$ de los casos se trataba del segundo ojo afectado (Fig. 1). El estudio contrastado entre dos grupos no mostró diferencias visuales estadísticamente significativas en ninguno de los tiempos valorados (Tabla 2).

\section{BIomicroscopía del CRISTALINO}

Los ojos fáquicos $(n=34)$ experimentaron una pérdida de 10.29 letras (SD, 4.22) y 18.97 letras (SD, 4.56) y los ojos intervenidos de cataratas $(n=13)$ una pérdida de 36.54 letras (SD, 8.13) y 53.08 letras (SD, 6.64) a los 3 y 5 años de seguimiento, respectivamente (Fig. 1). Se observó que los ojos de pacientes pseudofáquicos experimentaban una peor y significativa evolución visual que aquellos que conservaban el cristalino (Tabla 2).

\section{Discusión}

La indicación de la terapia con anti-VEGF en pacientes con DMAE exudativa está avalada por los ensayos clínicos realizados en los años 2005 y 2006, que demostraron la mejoría de la $\mathrm{AV}$ en pacientes con DMAE tratados con ranibizumab comparados con controles o terapia fotodinámica ${ }^{3,4}$. Posteriormente, numerosas publicaciones documentan los resultados de la terapia antiangiogénica en los dos primeros años de tratamiento, sin embargo, es escasa la información de la efectividad de este tratamiento más allá de 4 años de seguimiento, y aún más si la información se refiere a los resultados derivados de la práctica clínica real.

Nuestros datos sobre la efectividad del tratamiento anti-VEGF en la DMAE difieren de estudios previos, cuyos resultados muestran un mantenimiento o una discreta ganancia de AV a lo largo del tiempo. La ganancia o pérdida de letras debe ser interpretada según la AV inicial, ya que los sujetos con mejor AV al diagnóstico tienen menos probabilidad de ganancia de 
visión que aquellos que parten de una pobre $\mathrm{AV}$, por el efecto techo (Fig. 1). Por tanto, estas diferencias observadas podrían estar influidas porque la AV media inicial de nuestro estudio fue mayor que en estudios previos (Tabla 3).

También hay que considerar que los protocolos de tratamiento antiangiogénico seguidos por estudios como MARINA, ANCHOR y CATT se basaban en criterios rígidos y bien definidos con inyecciones fijas mensuales o bien un régimen PRN (PrONTO y CATT) que difieren de los que se pueden seguir en la práctica clínica real.

Cuando comparamos estudios retrospectivos de similar diseño, pero con un mayor tiempo de seguimiento, nuestros resultados son igualmente desfavorables. Los más representativos son el SEVEN UP ${ }^{15}$, que prolongó hasta 7 años el seguimiento de los pacientes incluidos en los ensayos ANCHOR, MARINA y HORIZON, y el CATT Study Follow ${ }^{16}$, con 5 años de seguimiento de los pacientes enrolados en el ensayo CATT. El balance positivo a favor de estos estudios puede ser reflejo del alto grado de seguimiento y tratamiento durante los dos primeros años del proceso neovascular cuando participaban en los estudios pivotales.

Además, todos estos estudios sufrieron pérdidas de muestra, siendo la causa más frecuente la falta de eficacia del tratamiento antiangiogénico, y estos pacientes no se contabilizaron en la valoración de efectividad terapéutica a largo plazo. Por tanto, los buenos resultados obtenidos pueden estar relacionados con este porcentaje de pacientes perdidos durante el seguimiento. Por el contrario, en nuestro estudio, todos los pacientes han completado un periodo de seguimiento de 5 años, independientemente de haber decidido no continuar con el tratamiento por desarrollo de atrofia geográfica o cicatriz disciforme.

Ganancias importantes de la AV pueden resultar poco relevantes si el resultado final es una visión pobre que impide al paciente la realización de las actividades de la vidacotidiana. Por ello, sería más correcto valorarla eficacia del tratamiento en términos de mantenimiento de la función visual. En estudios previos, el porcentaje de ojos que leen $>70$ letras se incrementa al final del periodo de seguimiento. En nuestro estudio, la proporción de ojos con AV inicial $\geq 70$ letras $(41.7 \%)$ fue disminuyendo a lo largo del tiempo, reduciéndose a la mitad (22.92\%) a los 3 años, y representó un 10.42\% de los casos al final del estudio (Tabla 4). Nuevamente consideramos que esta disparidad de resultados se debe al diseño del estudio realizado, al incluir a todos los pacientes durante un seguimiento de 5 años, independientemente de la eficacia del tratamiento.
Tabla 3. Agudeza visual basal y resultados visuales observados en diferentes estudios tras un año de seguimiento

\begin{tabular}{|l|c|c|}
\hline Estudio & AV media basal & $\begin{array}{c}\text { Balance medio tras 1 } \\
\text { año de seguimiento }\end{array}$ \\
\hline ANCHOR $^{4}$ & 47.1 & +11.3 \\
\hline MARINA $^{3}$ & 53.7 & +7.2 \\
\hline PrONTO $^{8}$ & 56.2 & +9.3 \\
\hline CATT $^{5}$ & 61.5 & +6.4 \\
\hline Nuestro estudio $^{-1.0}$ & 61.0 & -1.14 \\
\hline
\end{tabular}

A pesar de estos resultados, el comportamiento de la AV a lo largo del tiempo coincide con los estudios previos, esto es, los casos con una visión pobre basal experimentan una mejoría a corto plazo y los ojos con una mayor AV inicial, una menor ganancia neta inicial por el efecto techo.

En nuestro estudio, los casos que preservaron la AV (pérdida < 15 letras) a los 3 años fueron la mitad (50\%) y un tercio (33.3\%) a los 5 años. Estos resultados son marcadamente inferiores a los resultados de MARINA y ANCHOR, donde la estabilidad visual se alcanzó en el 95\% de los casos al año de seguimiento, y al estudio SEVEN UP, con un porcentaje del $66 \%$ tras 7 años de seguimiento. Por otro lado, no todos los pacientes perdieron progresivamente la visión, sino que un 14.6 y un $12.5 \%$ experimentaron una ganancia $>15$ letras a los 3 y 5 años de seguimiento, respectivamente.

En este estudio, el número de visitas anuales fue menor que en estudios previos, los cuales documentan 7-9 visitas en cada año de seguimiento. Esto se justifica porque, en muchos de nuestros pacientes, el número de revisiones médicas se fue espaciando progresivamente hasta un mínimo de dos visitas al año, ya que los ojos en estadios más avanzados, como la AV es muy baja debido al desarrollo de atrofia geográfica o cicatriz disciforme y la respuesta al tratamiento farmacológico es escasa o nula, no requieren un seguimiento tan exhaustivo. De la misma manera, el número medio de inyecciones en cada año de seguimiento fue menor al administrado en estudios recientemente publicados $^{20}$. Este hecho puede ser debido a que el abordaje clínico de los pacientes incluidos en este estudio fue anterior a la publicación de los actuales protocolos de tratamiento, lo que pudo determinar que se tratara de forma menos agresiva la enfermedad.

Los resultados sobre la influencia de distintas variables clínicas sobre la $\mathrm{AV}$ recogidas en este estudio son 
Tabla 4. Proporción de casos con buena función visual ( $\geq 70$ letras) al principio y al final de diferentes estudios, durante diferentes periodos de seguimiento.

\begin{tabular}{|c|c|c|c|}
\hline Estudio & Porcentaje inicial $\geq 70$ letras & Porcentaje final $\geq 70$ letras & Tiempo recogida de datos \\
\hline Talks, et al. ${ }^{17}$ & 16.4 & 33.7 & 1 aก̃o \\
\hline \multirow{2}{*}{$\begin{array}{l}\text { Writing Committee for de UK } \\
\text { Age-Related Macular Degeneration } \\
\text { EMR Users Group }\end{array}$} & \multirow{2}{*}{16} & 30 & 1 aก̃o \\
\hline & & 29 & 3 años \\
\hline Gillies, et al. ${ }^{19}$ & 23 & 37 & 3 años \\
\hline CATT Study Follow ${ }^{16}$ & 37.5 & 49.6 & 5 años \\
\hline SEVEN UP15 & 23 & 37 & 7 años \\
\hline \multirow[t]{2}{*}{ Nuestro estudio } & \multirow[t]{2}{*}{41.7} & 22.92 & 3 años \\
\hline & & 10.42 & 5 años \\
\hline
\end{tabular}

concordantes con estudios previos: los pacientes $>75$ años presentan un curso más acelerado del proceso neovascular21,22; los cambios de AV a lo largo del tiempo no están influenciados por las diferencias de género ${ }^{21,22 ;}$; los segundos ojos mantienen mayores AV que los primeros ojos en todos los tiempos de seguimiento, aunque no de forma significativa ${ }^{17,23,24}$.

Por último, observamos que, a pesar de una mayor AV inicial en los ojos pseudofáquicos, estos experimentaron un deterioro visual más acusado, tres veces mayor que los ojos fáquicos y un resultado visual final más desfavorable que estos últimos, a pesar de las limitaciones visuales que implica la presencia de una catarata avanzada propia de una población envejecida. Nuestros datos contrastan con estudios previos en los que no encuentran diferencias estadísticamente significativas entre estos dos grupos $^{25-27}$, si bien es cierto, que alguno de ellos sugiere que los ojos fáquicos tienden a mostrar una menor pérdida de AV. Para explicar estas diferencias, se ha propuesto la hipótesis de que la cirugía de catarata podría condicionar alteraciones en la farmacodinámica y/o farmacocinética del medicamento liberado en la cavidad vítrea. Por un lado, se han descrito cambios en la composición proteica del humor vítreo, así como una mayor incidencia de desprendimiento de vítreo posterior tras esta cirugía, que podrían influir en la vida media del fármaco. Por otro lado, el estado de ojo pseudofáquico podría favorecer el aclaramiento del fármaco a través de las vías de eliminación del humor acuoso en la cámara anterior.

\section{Conclusiones}

Las evidencias actuales muestran la necesidad de un mínimo de siete inyecciones el primer año de tratamiento. Nuestro estudio se desarrolla en un periodo en el que no estaba claramente definida la estabilidad de la lesión en función del protocolo utilizado (mensual, PRN, T\&E), por lo que nuestros pacientes en el periodo estudiado estarían infratratados. Los resultados visuales también estarían influenciados al incluir en el análisis de AV a los ojos con desarrollo de atrofia geográfica o cicatriz disciforme.

\section{Conflicto de intereses}

Los autores declaran no tener conflicto de intereses.

\section{Responsabilidades éticas}

Protección de personas y animales. Los autores declaran que para esta investigación no se han realizado experimentos en seres humanos ni en animales.

Confidencialidad de los datos. Los autores declaran que han seguido los protocolos de su centro de trabajo sobre la publicación de datos de pacientes.

Derecho a la privacidad y consentimiento informado. Los autores declaran que en este artículo no tomaron datos de pacientes al ser un estudio retrospectivo.

\section{Bibliografía}

\footnotetext{
1. Ferris FL, Wilkinson CP, Bird A, Chakravarthy U, Chew E, Csaky K, et al. Clinical Classification of Age-related Macular Degeneration. Ophthalmology. 2013;120:844-51.

2. Mordenti J, Cuthbertson RA, Ferrara N, Thomsen K, Berleau L, Licko V, et al. Comparisons of the intraocular tissue distribution, pharmacokinetics, and safety of $125 \mathrm{I}$-labeled full-length and Fab antibodies in rhesus monkeys following intravitreal administration. Toxicol Pathol. 1999;27:536-44
} 
3. Rosenfield PJ, Brown DM, Heier JS, et al. Ranibizumab for neovascular age-related macular degeneration. N Engl J Med. 2006;355:1419-31.

4. Brown DM, Kaiser PK, Michels M, Soubrane G, Heier JS, Kim RY, et al Ranibizumab versus verteporfin for neovascular age-related macular degeneration. N Engl J Med. 2006;355:1432-44

5. The CATT Research Group. Ranibizumab and bevacizumab for neovascular age-related macular degeneration. N Engl J Med 2011; 364: 1897-908.

6. Regillo CD, Brown DM, Abraham P, Yue H, lanchulev T, Schneider S, et al. Randomized, double-masked, sham-controlled trial of ranibizumab for neovascular age-related macular degeneration: PIER Study year 1. Am J Ophthalmol. 2008; 145:239-48.

7. Schmidt-Erfurth U, Eldem B, Guymer R, Korobelnik JF, Schlingemann RO, Axer-Siegel R, et al. Efficacy and safety of monthly versus quarterly ranibizumab treatment in neovascular age-related macular degeneration: the EXCITE study. Ophthalmology. 2011;118:831-9.

8. Fung AE, Lalwani GA, Rosenfeld PJ, Dubovy SR, Michels S, Feuer WJ, et al. An Optical Coherence Tomography-Guided, Variable Dosing Regimen with Intravitreal Ranibizumab (Lucentis) for Neovascular Age-Related Macular Degeneration. Am J Ophthalmol. 2007;143:566-83.

9. Boyer DS, Heier JS, Brown DM, Francom SF, lanchulev T, Rubio RG. A Phase IIlb study to evaluate the safety of ranibizumab in subjects with neovascular age-related macular degeneration. Ophthalmology. 2009; 116:1731-9

10. Sadda SR. HORIZON extension trial of ranibizumab [LUCENTIS@] for neovascular agerelated macular degeneration [AMD]: first-year safety and efficacy results. Retina Society Annual Meeting. Scottsdale, USA, 2008.

11. Holz FG, Amoaku W, Donate J, Guymer RH, Kellner U, Schlingemann RO, et al. Safety and efficacy of a flexible dosing regimen of ranibizumab in neovascular age-related macular degeneration: the SUSTAIN study. Ophthalmology. 2011; 118:663-71.

12. Spaide R. Ranibizumab according to need: a treatment for age-related macular degeneration. Am J Ophthalmol. 2007; 143:679-80.

13. Browning DJ, Kaiser PK, Rosenfeld PJ, Stewart MW. Aflibercept for age-related macular degeneration: a game-changer or quiet addition? Am J Ophthalmol. 2012;154:222-6

14. Stewart MW. Clinical and differential utility of VEGF inhibitors in wet age-related macular degeneration: focus on aflibercept. Clin Ophthalmol. 2012;6:1175-186.

15. Rofagha S, Bhisitkul RB, Boyer DS, Sadda SR, Zhang K, SevenUp Study Group. Seven-year outcomes in ranibizumab-treated patients in ANCHOR, MARINA and HORIZON: a multicenter cohort study (SEVEN-UP). Ophthalmology 2013;120(11):2292-9.
16. Comparison of Age-Related Macular Degeneration Treatment Trials (CATT) Research Group. Five-Year Outcomes with Anti-Vascular Endothelial Growth Factor Treatment of Neovascular Age-Related Macular Degeneration. Ophthalmology. 2016;123:1751-61.

17. Talks JS, Lotery AJ, Ghanchi F, Sivaprasad S, Johnston RL, Patel N, et al. First-Year Visual Acuity Outcomes of Providing Aflibercept According to de VIEW Study Protocol for Age-Related Macular Degeneration. Ophthalmology. 2016;123:337-43.

18. Committee for de UK Age-Related Macular Degeneration EMR Users Group. The neovascular age-related macular degeneration database: a multicenter study of 92976 ranibizumab injections: report 1: visual acuity. Ophthalmology. 2014;121:1092-101.

19. Gilles MC, Campain A, Barthelmes D, Simpson JM, Arnold JJ, Guymer RH, et al. Long-Term Outcomes of Treatment of Neovascular Age-Related Macular Degeneration. Ophthalmology. 2015; 122:1837-45.

20. Gemenetzi M and Patel PJ. A Systematic Review of the Treat and Extend Treatment Regimen with Anti-VEGF Agents for Neovascular Age-Related Macular Degeneration. Ophthalmol Ther. 2107;6:79-92.

21. Kaiser PK, Brown DM, Zhang K, Hudson HL, Holz FG, Shapiro H, et al. Ranibizumab for predominantly classic neovascular age-related macular degeneration: subgroup analysis of first-year ANCHOR results. Am J Ophthalmol. 2007;144(6):850-7.

22. Boyer DS, Antoszyk AN, Awh CC, Bhisitkul RB, Shapiro H, Acharya NR, et al. MARINA Study Group. Subgroup analysis of the MARINA study of ranibizumab in neovascular age-related macular degeneration. Ophthalmology. 2007; 114:246-52.

23. Zarranz-Ventura J, Liew G, Johnson RL, Xing W, Akerele T, McKibbin M, et al. The Neovascular Age-Related Macular Degeneration Database. Report 2: Incidence, Management, and Visual Outcomes of Second Treated Eyes. Ophthalmology. 2014; 121:1966-75.

24. Eldaly MA, Styles C. First versus second eye intravitreal ranibizumab therapy for wet AMD. Retina. 2009;29:325-8.

25. Weinberg DV, Shapiro H, Ehrlich J. Ranibizumab Treatment Outcome in Phakic versus Pseudophakic Eyes. An individual Patient Data Analysis of 2 Phase 3 Trials. Ophthalmology. 2013;120:1278-82.

26. Baek JS, Cho HJ, Cho SW, Kim CG, Kim JW. Intravitreal ranibizumab injection for neovascular age-related macular degeneration in phakic versus pseudophakic eyes. Retina. 2013;33:467-73.

27. Ozkaya A, Alkin Z, Yazici A, Demirok A. Comparison of intravitreal ranibizumab in phakic and pseudophakic neovascular age-related macular degeneration patients with good baseline visual acuity. Retina. 2014;34:853-9. 